Relations industrielles

Industrial Relations

\title{
"It Was Never Easy ", Edité par The Canadian Brotherhood of Railway, Transport and General Workers, Mutual Press Limited, Ottawa, 1961, 414 pages.
}

\section{Louis-Marie Tremblay}

Volume 18, numéro 1, janvier 1963

URI : https://id.erudit.org/iderudit/1021466ar

DOI : https://doi.org/10.7202/1021466ar

Aller au sommaire du numéro

\section{Éditeur(s)}

Département des relations industrielles de l’Université Laval

ISSN

0034-379X (imprimé)

1703-8138 (numérique)

Découvrir la revue

Citer ce compte rendu

Tremblay, L.-M. (1963). Compte rendu de [«It Was Never Easy », Edité par The Canadian Brotherhood of Railway, Transport and General Workers, Mutual Press Limited, Ottawa, 1961, 414 pages.] Relations industrielles / Industrial Relations, 18(1), 131-132. https://doi.org/10.7202/1021466ar

Tous droits réservés (C Département des relations industrielles de l’Université Laval, 1963
Ce document est protégé par la loi sur le droit d'auteur. L'utilisation des services d'Érudit (y compris la reproduction) est assujettie à sa politique d'utilisation que vous pouvez consulter en ligne.

https://apropos.erudit.org/fr/usagers/politique-dutilisation/ 
était mise sur l'aspect historique plutôt que l'aspect statistique. Pour celui qui s'intéresse à l'histoire ouvrière, cette première période s'avère une précieuse source d'information.

L'annuaire subit ensuite une première modification. Tout en conservant un bref résumé des évènements de l'année, on consacrait une large part aux données statistiques. Malgré certaines lacunes, le rapport s'avérait très utile pour l'écologie du syndicalisme. Mais au lieu de s'améliorer sur ce point, il s'est depuis quelques années constamment détérioré. Alors que la plupart des publications du gouvernement fédéral s'efforcent de fournir des statistiques de plus en plus nombreuses, détaillées et diversifiées, celleci consacre de moins en moins d'espace à ce genre de renseignement. Elle ne contient que quatre tableaux. Cette situation dure depuis 1959 alors que l'on a fait disparaître, entre autres, deux informations dès plus importantes; la répartition des unions locales et des effectifs par industrie ainsi que la répartition par province. C'est pourquoi, il n'est pas surprenant que cetto publication du Ministère fédéral du travail prenne de plus en plus l'allure d'un simple bottin des organisations syndicales.

Nous regrettons que \& Labour Organisations in Canada $\gg$ qui constituait, jusqu'à quelques années, une bonne source d'information, devienne un instrument de moins en moins utile sur le plan académique. Ceci est d'autant plus déplorable que le ministère possède des données qu'il ne publie pas, mais qu'il fournit sur demande. Nous comprenons que les renseignements recueillis par la division de la recherche comportent une certaine marge d'erreurs. Cependant, avec les restrictions appropriées, cela est préférable à rien du tout. D'ailleurs, ce ne sont pas les seules statistiques fédérales qui contierinent une marge d'erreurs. Souhaitons en terminant que le ministère, en rafinant ses instruments de collection de statistiques, modifie sa politique actuelle.

L.-M. TREMBLAY

\& It Was Never Easy \$, Edité par The Canadian Brotherhood of Railway, Transport and General Workers, Mu- tual Press Limited, Ottawa, 1961, 414 pages.

C'est l'histoire de la Fraternité canadienne des employés de chemin de fer, du transport et autres employés, mais une histoire vue sous un angle particulier, soit celui des luttes et conflits que la fraternité a connus depuis sa formation en 1908. Le manuscrit original est l'oeuvre de W.E. Greening. M. M. MacLean a complété celui-ci et écrit le texte final. Ce dernier connaît bien le sujet car il a été l'un des principaux artisans de cette organisation ouvrière en qualité de secrétairetrésorier et d'éditeur de la revue de la fraternité jusqu'en 1942 où il passait au service du Ministère du travail fédéral. Il a donc non seulement vécu, mais été l'un des principaux participants, de la plupart des évènements qui sont racontés dans ce volume. C'est pourquoi, on attend beaucoup de sa participation. Mais, la lecture de * It was Never Easy » est à la fois intéressante et décevante.

La C.B.R.T. (à l'origine, C.B.R.E.) est une expérience syndicale typiquement canadienne qui prit naissance dans les provinces maritimes. Elle fut un temps, comme l'O.B.U., une internationale à rebours, en recrutant des membres aux Etats-Ûnis. Elle a joué un rôle important dans le Congrès pancanadien du travail et le Congrès canadien du travail ainsi que dans l'établissement de relations et de liens entre le parti C.C.F. et le mouvement ouvrier. L'emphase délibérément accordée aux difficultées externes et internes de la C.B.R.T. ne permet pas de faire ressortir suffisamment la progression et l'évolution de cette organisation. Le rôle joué par la fraternité dans le Congrès pancanadien du travail est sous-développé. Nous aurions aimé aussi que tranche davantage et de façon plus précise la personalité de Aaron Mosher fondateur et inspirateur de la fraternité dont il fut président jusqu'en 1952. Il cumula la charge de président dans le Congrès pancanadien du travail de 1927 à 1940 et dans le Congrès du Travail du Canada. Il servit aussi, pendant la guerre dans les commissions consultatives gouvernementales. Mosher a été et demeurera l'une des figures dominantes de l'histoire syndicale au Canada. 
«It Was Never Easy 》 est décevant par sa facture, l'organisation du matériel et le peu d'application des principes de la méthodologie scientifique. Sur ce point, cette monographie fait pâle figure avec la série publiée par John Wiley and Sons sous la direction de Walter Galenson. Son caractère anecdotique limite le nombre de lecteurs qui en dehors de la C.B.R.T. peuvent être atteints avec intérêt. Nous croyons qu'une telle faiblesse pourrait être évitée si les organismes ouvriers, en de semblables occasions, faisaient appel à du personnel du monde académique. La collaboration entre des practiciens et des hommes de science s'avèrerait plus rentable, les premiers fournissant leurs expériences personnelles et leurs connaissances intimes des faits, les seconds apportant la méthode scientifique. Ainsi, une monographie historique ne se résumerait pas dans une simple collection de faits car ces derniers pourraient être replacés dans leur contexte historique suivant une perspective appropriée ce qui permettrait d'en tirer des conclusions valables et utiles.

L.-M. TREMBLAY

Socialisation et Personne humaine. Compte rendu de la $47 \mathrm{e}$ semaine sosiale de France, Grenoble 1960. 1 vol. 434 pages. Chronique sociale de France, 16, rue du Plat, Lyon.

L'encyclique Mater et Magistra et la Déclaration de l'Episcopat canadien à l'occasion de la fête du travail ont contribué à attirer l'attention du public de notre pays sur le phénomène important de la socialisation. A la suite du dernier document collectif des évêques, plusieurs groupements ont mis ce sujet à leur programme d'études. Et, de part et d'autre, on nous demande de la bibliographie. Or, l'ouvrage fondamental, qui n'a pas été étranger à ce que l'on retrouve dans Mater et Magistra, est le compte rendu des travaux présentés à la Semaine sociale de France de 1960, laquelle portait sur socialisation et Personne humaine $\rightarrow$. D'ailleurs, on notera mot à mot dans l'encyclique des passages de la lettre adressée par le cardinal Tardini au président de la Semaine.
Voici les principales communications: Le mouvement de socialisation: risques et chances (Alain Barrère); Nouvelles formes et nouveau style de la vie sociale (René Théry); Progrès des techniques et volonté de rationalisation (Robert Goetz-Girey); L'évolution des classes et des structures sociales (Francis Closon); Enrichissement et asservissement de la personne (Joseph Folliet); Le retard des institutions (J.-L. Quermonne); Socialisation et tendances totalitaires (J.-Y. Calvez); Crise des socialismes (René Remond); La conscience du social et le personnalisme (Yvon Bres); Perspectives chrétiennes sur la vie personnelle et la vie collective (Yves Congar); La participation des personnes et des groupes à la vie sociale (R. P. Thomas); Le public et le privé (Jean Lacroix); Sciences humaines et techniques d'action sur l'homme (Georges Hahn); Le droit au service de la personne (René Savatier); La contrainte et la répression (Albert Chavanne); La participation à la vie des communautés locales (Henri Théry); Economie concertée et démocratie économique (Eugène Descamps); Vers de nouvelles structures politiques (Jean Rivero). Cette simple énumération des titres avec le nom des auteurs suffit pour montrer tout l'intérêt qu'il y a à prendre connaissance de cet ouvrage. On peut se le procurer aux Presses de l'Université Laval, C. P. 999, Québec.

The Law of Labour Relations in Sweden, par Folke Schmidt; Harvard University Press, Cambridge, Massachussetts, 1962, publié au Canada par S.S. Reginalds Saunders and Company Ltd, Toronto, 343 pp.

Le système de relations industrielles suédois est sûrement lun des plus intéressants à connaître dans le cadre des études portant sur les systèmes comparés de relations du travail. En plusieurs de ses institutions il manifeste une originalité qui, Norvège et Danemark mis à part, le distingue de ceux des autres pays occidentaux. En Suède, plus de $90 \%$ des travailleurs industriels salariés sont membres de syndicats libres, volontaires. Ces derniers se comportent d'une façon démocratique et sont libres de toute corruption dans la conduite de leurs affaires. Il n'est pas un pays démocratique où l'appartenance syndicale libre soit élevée d ce point et où 